\title{
High-Frequency Chest Wall Compression Therapy in Neurologically Impaired Children
}

\author{
Kathryn Fitzgerald MSN CPNP, Jessica Dugre MSN, Sobhan Pagala, Peter Homel PhD, \\ Michael Marcus MD, and Mikhail Kazachkov MD
}

\begin{abstract}
BACKGROUND: Children with neurological impairment often suffer from insufficient airway secretion clearance, which substantially increases their respiratory morbidity. The goal of the study was to assess the clinical feasibility of high-frequency chest wall compression (HFCWC) therapy in neurologically impaired children with respiratory symptoms. METHODS: This was a single-center, investigator initiated, prospective study. Twenty-two subjects were studied for 12 months before and 12 months after initiation of HFCWC therapy, and 15 subjects were followed for an additional 12 months. The threshold of adherence to HFCWC therapy was $70 \%$. The number of pulmonary exacerbations that required hospitalization was recorded. RESULTS: Forty-five percent of the subjects required hospital admission before initiation of HFCWC therapy. This rate decreased to $36 \%$ after the first year with HFCWC, and to $13 \%$ after the second year with HFCWC. There was a statistically significant reduction of the number of hospital days at follow-up, relative to before treatment. Use of an assisted-cough device or the presence of tracheostomy did not significantly affect hospitalization days. CONCLUSIONS: Regular HFCWC therapy may reduce the number of hospitalizations in neurologically impaired children. Key words: neurological impairment; secretion clearance; respiratory; high-frequency chest wall compression; hospitalization. [Respir Care 2014;59(1): 107-112. () 2014 Daedalus Enterprises]
\end{abstract}

\section{Introduction}

Respiratory management of children with neurological impairment is complex. ${ }^{1,2}$ Upper-airway obstruction syn-

\footnotetext{
At the time of this study, Ms Fitzgerald, Ms Dugre, and Dr Kazachov were affiliated with the Division of Pediatric Pulmonology, Maimonides Infants and Children's Hospital, Brooklyn, New York, but are now affiliated with the Division of Pediatric Pulmonology, New York University Langone Medical Center, New York, New York. Mr Pagala and Dr Marcus are affiliated with the Division of Pediatric Pulmonology, Maimonides Infants and Children's Hospital, Brooklyn, New York. Dr Homel is affiliated with the Office of Research Administration, Maimonides Medical Center, Brooklyn, New York.
}

This research was partly supported and monitored by Hill-Rom Advanced Respiratory, which provided funding and vest generators, but did not participate directly in the data collection and analysis. Drs Fitzgerald and Kazachkov have disclosed relationships with Hill-Rom. The authors have disclosed no other conflicts of interest.

Ms Fitzgerald presented a version of this paper at the International Conference of the American Thoracic Society, held May 13-18, 2011, in Denver, Colorado. dromes, reflux and aspiration of gastric contents, impaired lower-respiratory secretion clearance due to weak cough, frequent lower-respiratory-tract infections, chest wall deformities and related deformities of the tracheobronchial tree contribute to morbidity and pose a constant challenge to clinicians. ${ }^{3-6}$ Frequent lower-respiratory-tract infections require multiple courses of antibiotics and hospital and ICU admissions that heavily burden the healthcare system. . $^{7-9}$

Impaired clearance of lower-respiratory secretions leads to acute and chronic lower-respiratory-tract infection, exacerbating a build-up of lower-respiratory secretions. ${ }^{10,11}$ In an attempt to promote clearance of these lowerrespiratory secretions, chest physical therapy is often incorporated in the management of children with neuro-

Correspondence: Kathryn Fitzgerald MSN CPNP, Division of Pediatric
Pulmonology, New York University Langone Medical Center, 550 First
Avenue, New York NY 10016. E-mail: Kathryn.Fitzgerald@nyumc.org.

DOI: $10.4187 /$ respcare. 02446 


\section{High-Frequency Chest Wall Compression Therapy in Neurologically ImPaired Children}

logical impairment. ${ }^{1,4,12}$ An appreciation of the role of impaired cough clearance in the respiratory morbidity of children with neuromuscular weakness led to widespread use of the cough-assistance device (mechanical insufflator-exsufflator), which was shown to be effective and now is strongly recommended as a standard of care in these children. ${ }^{13-18}$ However, use of the cough-assistance device in children with cerebral palsy and other neurological syndromes has not yet been studied, is controversial, and the implementation differs between institutions.

Intrapulmonary percussive ventilation therapy was shown to be effective in adolescents and young adults with neuromuscular disease. ${ }^{19}$ Other chest physical therapy modalities include end-expiratory positive pressure devices, manual chest physical therapy, and high-frequency chest wall compression (HFCWC). The selection of chest physical therapy methods used in this group of patients depends on several factors, including the cognitive status of the patient, severity of respiratory compromise, and in many ways on the clinical experience of the clinicians.

There are limited data on the efficacy of HFCWC therapy in children with neurological impairment, and no data on the impact of HFCWC on healthcare costs in this category of patients. We conducted a prospective study to evaluate the clinical feasibility of HFCWC in children with neurological impairment and respiratory symptoms. We hypothesized that HFCWC would decrease respiratory morbidity in this population of children. We compared the number of hospitalizations and hospital days in a group of children with neurological impairment and respiratory symptoms before and after the introduction of HFCWC therapy.

\section{Methods}

This was a single-center, investigator initiated, prospective study assessing the efficacy of HFCWC therapy in patients with severe neurological impairment and frequent pulmonary exacerbations. The research was conducted at Maimonides Medical Center, Brooklyn, New York. Our institutional review board approved the study. The parents of all study subjects gave informed consent.

\section{Initial Subject Enrollment}

The inclusion criteria were age $>18$ months, chest circumference $>48 \mathrm{~cm}$, a diagnosis of neurological impairment, and $\geq 2$ lower-respiratory-tract infections that required supplemental oxygen and hospitalization in the 12 months prior to study enrollment. The exclusion criteria were diagnosed allergy with a respiratory trigger, unstable head or spinal injury, unresolved pneumothorax or pneumomediastinum, unresolved hemorrhage, hypotension requiring vasopressors or positioning, bronchopleural fis-

\section{QUICK LOOK}

\section{Current knowledge}

Children with neurological impairment frequently suffer from insufficient airway secretion clearance, which increases respiratory morbidity. Various airway clearance techniques in these patients have varying levels of success.

\section{What this paper contributes to our knowledge}

The addition of high-frequency chest wall compressions (with The Vest) to an airway clearance regimen in children with neurologic impairment was associated with fewer hospitalizations.

tula, gross hemoptysis, pulmonary embolism, burns, open wounds or skin infections on the thorax, osteomyelitis of the ribs, and osteoporosis with a history of fractures.

\section{Follow-Up Subject Enrollment}

Chart review was conducted 12 months after the cessation of the main study. Study subjects who completed the main study and remained adherent to HFCWC therapy were considered for enrollment in the follow-up study. Subjects were re-consented for the follow-up study, and data collection was performed retrospectively.

\section{Study Procedures}

A complete physical assessment was performed at study enrollment, every 3 months during the study, and at the final visit. Data (Table 1) were collected during study visits and telephone interviews, which were conducted every 14 days for 1 year after enrollment. Subjects continued their routine care, which may have included use of the cough-assist device (CoughAssist, Respironics, Murrysville, Pennsylvania), suctioning, and anti-reflux, antiseizure, and other medications as usual.

HFCWC therapy was initialized upon study enrollment. Once enrolled, a home visit was made for device fitting and training the subject and caregivers on the HFCWC device (The Vest, model 105, Hill-Rom, St Paul, Minnesota). The Vest has a built-in hour-meter to measure adherence. HFCWC therapy was used for 12 months, 20 min twice daily, and increased up to 4 times a day for lower-respiratory-tract infection associated with increased oxygen requirement. Subjects $18-36$ months old used a frequency of $10 \mathrm{~Hz}$, and subjects $>36$ months old used a frequency of $12 \mathrm{~Hz}$. All the subjects used an inflation pressure of 3 arbitrary units. The threshold for adherence 
Table 1. Data Assessment

\begin{tabular}{lcccc}
\hline \hline \multicolumn{1}{c}{ Assessment } & Baseline & Monthly & $\begin{array}{c}\text { As } \\
\text { Occurred }\end{array}$ & $\begin{array}{c}\text { Study } \\
\text { Exit }\end{array}$ \\
\hline Eligibility data & $\mathrm{X}$ & & & \\
Demographic data & $\mathrm{X}$ & & & \\
Medical history & $\mathrm{X}$ & & & \\
Vest hour-meter readings & & $\mathrm{X}$ & & $\mathrm{X}$ \\
Hospitalizations & & $\mathrm{X}$ & $\mathrm{X}$ \\
Antibiotics & & $\mathrm{X}$ & $\mathrm{X}$ \\
Clinic visits & & $\mathrm{X}$ & $\mathrm{X}$ \\
Adverse events & & $\mathrm{X}$ & $\mathrm{X}$ \\
Protocol deviations & & & $\mathrm{X}$ & $\mathrm{X}$ \\
\hline
\end{tabular}

to HFCWC therapy was $70 \%$. Subjects who were nonadherent were not permitted to continue in the study, and their HFCWC devices were picked up and returned to Hill-Rom immediately after non-adherence was identified.

A 6 month wash-in period began at HFCWC initiation. The duration of the wash-in period reflected the suggestion that clinical benefits from HFCWC become more apparent after 6 months of treatment. ${ }^{19}$ The data from the wash-in period were excluded from analysis. An equivalent 6-month period before treatment was also excluded from data analysis, so that no seasonal difference skewed the data. A total of 12 months of data were analyzed for each subject (6 months before initiation of therapy and 6 months after). For example, for a subject enrolled on $6 / 1 / 2009$, data from 1/1/2009-6/31/2009 and 1/1/2010$6 / 31 / 2010$ were analyzed and compared. Equivalent 6-month periods before and after interventions were used, so that no seasonal differences skewed the data. The study timeline is summarized in the Figure.

\section{Follow-up Study Procedures}

The study subjects were followed for 12 months after completion of the main study. The data from the last 6 months of HFCWC therapy were compared with those collected during the corresponding 6 months before therapy initiation. An equivalent 6-month period of data from the 1-year follow-up was compared with the previously collected data from the corresponding 6-month period before enrollment.

\section{Statistical Analysis}

Normally distributed data are given as mean $\pm \mathrm{SD}$, skewed continuous variables as median and IQR, and categorical variables (eg, sex) as number and percent. More than $50 \%$ of the subjects had no hospital days (recorded as 0 ), and so this outcome was dichotomized and summarized in terms of percent hospitalization (yes/no). A one-

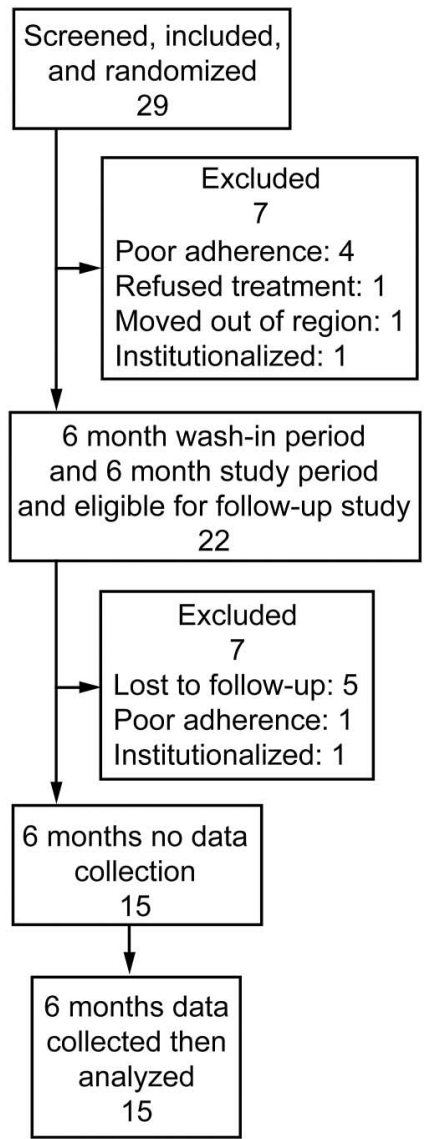

Figure. Flow chart of the study timeline.

sample chi-square test was used to test for differences in the proportion of subjects with different sex, race, diagnoses, technology modalities, and comorbid conditions between the main-study subjects and the follow-up subjects. The age difference between the main-study and follow-up subjects was assessed with a one-sample $t$ test.

Primary outcome data were analyzed using general linear modeling, which allows for repeated-measures analysis of subjects with missing data, under the assumption that the data are missing at random, so that all subjects were included for analysis. An underlying gamma distribution for skewed outcomes was assumed, in order to compare the number of hospital days across time (ie, before vs after HFCWC treatment and follow-up). The possible impact of the CoughAssist device and tracheostomy upon outcome measures was analyzed by including appropriate group $\times$ time interactions in the general linear modeling analysis. Data were analyzed with statistics software (SAS 9.1, SAS Institute, Cary, North Carolina) for the general linear modeling. All analyses assumed differences were significant at $P<.05$.

Based on Giarraffa et $\mathrm{al}^{20}$ and Plioplys et al, ${ }^{21}$ the effect size for reduction in the number of hospital days was initially estimated to be between 1.36 and 1.55 days. Since 


\section{High-Frequency Chest Wall Compression Therapy in Neurologically ImPaired Children}

Table 2. Demographics and Clinical Characteristics of Subjects Who Received High-Frequency Chest Wall Compressions Therapy

\begin{tabular}{|c|c|c|c|}
\hline & $\begin{array}{c}\text { Main } \\
\text { Study } \\
n=22\end{array}$ & $\begin{array}{c}\text { Follow-up } \\
\text { Study } \\
n=15\end{array}$ & $P$ \\
\hline Age, mean $\pm \mathrm{SD}$ y & $9.55 \pm 5.57$ & $9.44 \pm 5.49$ & .95 \\
\hline Male & $14(64)$ & $8(53)$ & .58 \\
\hline Female & $8(36)$ & $7(47)$ & \\
\hline African American & $5(23)$ & $2(13)$ & .84 \\
\hline Asian & $3(14)$ & $2(13)$ & \\
\hline White & $8(36)$ & $6(40)$ & \\
\hline Hispanic & $6(27)$ & $5(33)$ & \\
\hline \multicolumn{4}{|l|}{ Diagnosis } \\
\hline Cerebral palsy & $14(64)$ & $9(60)$ & .47 \\
\hline Brain malformation & $4(18)$ & $3(20)$ & .52 \\
\hline Static encephalopathy & $1(5)$ & $1(7)$ & .54 \\
\hline Myopathy & $1(5)$ & $0(0)$ & .46 \\
\hline Neurodegenerative disease & $2(9)$ & $2(13)$ & .40 \\
\hline \multicolumn{4}{|l|}{ Treatments } \\
\hline Bi-level positive airway pressure & $6(27)$ & $3(20)$ & .73 \\
\hline Assisted cough & $12(55)$ & $9(60)$ & .86 \\
\hline Ventilator & $4(18)$ & $2(13)$ & .60 \\
\hline Oxygen & $9(41)$ & $7(47)$ & .84 \\
\hline Gastrostomy tube & $18(82)$ & $12(80)$ & $>.99$ \\
\hline Tracheostomy & $6(27)$ & $3(20)$ & .73 \\
\hline \multicolumn{4}{|l|}{ Comorbidities } \\
\hline Gastroesophageal reflux disease & $16(73)$ & $11(73)$ & $>.99$ \\
\hline Seizure disorder & $14(64)$ & $10(67)$ & $>.99$ \\
\hline Scoliosis & $16(73)$ & $9(60)$ & .42 \\
\hline
\end{tabular}

Values are number $(\%)$ unless otherwise indicated.

these estimates appeared to be rather high, the lower one was reduced by half, to 0.68 . Based on that effect size, a minimum of 19 subjects was assumed to be needed to detect a paired difference of that magnitude, with an alpha of .05 and power of $80 \%$. Assuming a $25 \%$ drop-out rate, the minimum sample size for recruitment was determined to be 25 .

\section{Results}

Twenty-nine subjects consented to inclusion in the main study. Four subjects were withdrawn due to non-adherence to HFCWC, one subject refused treatment, one subject moved out of state, and one subject was institutionalized. Twenty-two subjects completed the main study. The mean \pm SD age of the main study subjects was $9.55 \pm 5.57$ years. Sixty-four percent of the subjects had cerebral palsy, and $45 \%$ had other diagnoses. Forty-five percent were on mechanical ventilation, and $27 \%$ had tracheostomy (Table 2).

Of the 22 subjects who completed the main study, 15 were included in the follow-up group, 7 of whom were
Table 3. Rate of Hospital Admissions Before and After Initiation of High-Frequency Chest Wall Compressions Treatment and at Follow-up

\begin{tabular}{lcrc}
\hline \hline & \multicolumn{3}{c}{ Hospitalized } \\
\cline { 2 - 3 } & No & Yes & \\
\hline Before, $n=22$ & $12(55)$ & $10(45)$ & NA \\
After, $n=22$ & $14(64)$ & $8(36)$ & .47 \\
At follow-up, $n=15$ & $13(87)$ & $2(13)$ & .002 \\
\hline
\end{tabular}

later excluded (5 lost to follow-up, one institutionalized, and one was non-adherent to HFCWC during the follow-up period). The mean baseline age of the follow-up subjects was $9.44 \pm 5.49$ years. There were no significant differences in demographics, diagnoses, or technology modalities between the main-study subjects and the follow-up study subjects (see Table 2).

There was a statistically significant reduction in the number of hospital days at follow-up, relative to before treatment $(P=.03)$, due to a dramatic decrease in the rate of hospitalization at follow-up (Table 3). Forty-five percent of the subjects required hospital admission before initiation of HFCWC. The rate of hospitalization decreased to $36 \%$ after the first year of using HFCWC $(P=.47)$, and to $13 \%$ at follow-up $(P=.002)$. The use of CoughAssist or the presence of tracheostomy did not significantly affect hospitalization days $(P=.50$, and $P=.17$, respectively).

\section{Discussion}

There are limited data on HFCWC in children with neurological impairment in the peer-reviewed literature. One study found that 12 months of HFCWC significantly decreased the number of pneumonias in subjects with cerebral palsy; however, the sample size was only 7 subjects. ${ }^{21}$ Yuan et al ${ }^{22}$ found a trend toward fewer hospitalizations and less intravenous antibiotic use with HFCWC in a randomized controlled trial with 23 subjects with cerebral palsy and neuromuscular disorders.

In our subjects HFCWC reduced the number of hospitalizations and hospital days during the second year of using HFCWC, which suggests that prolonged use of HFCWC benefits children with neurological impairment and respiratory disorders. This finding concurs with Warwick et $\mathrm{al}^{23}$ who found that long-term (7-26 months) of HFCWC significantly changed toward positivity the negative slope of lung function parameters in children with cystic fibrosis.

Patients with neurological impairment have multiple factors that predispose them to the retention of respiratory secretions and bronchiectasis, ${ }^{24-26}$ which leads to the characteristic thick and tenacious respiratory secretions. 


\section{High-Frequency Chest Wall Compression Therapy in Neurologically ImPaired Children}

Plioplys et $\mathrm{al}^{21}$ studied HFCWC in children with neurological impairment and cerebral palsy and found that HFCWC dramatically increased effective suctioning, defined as suctioning attempts that retrieved sputum (summation score of effective suctioning 4,825 before treatment, versus 10,445 after treatment, $P=.008$ ). It is possible that HFCWC benefits children with cerebral palsy and neurological impairment by promoting the mobilization of tracheobronchial secretions and facilitating mucociliary clearance, as it does in children with cystic fibrosis. ${ }^{27}$

Impaired cough is a major contributor to respiratory morbidity. ${ }^{2,12}$ Cough assistance benefits and is now recommended for children with congenital muscle dystrophies. ${ }^{14}$ In our study the use of the CoughAssist device did not significantly affect hospitalization days, which raises the question of whether improving the rheological characteristics of airway secretions provides clinical benefit even in the absence of effective cough. It is possible that HFCWC loosens lower-respiratory secretions sufficiently to improve mucociliary clearance, but this hypothesis needs to be considered with caution. Acute respiratory deterioration after HFCWC occurred in a child with cerebral palsy, and was attributed to airway secretions mobilization in the presence of ineffective cough. ${ }^{28}$ Those authors suggested that cough adequacy should be determined before initiating secretion mobilization treatments in such children. Since we did not assess cough adequacy in our subjects, we cannot make a recommendation on assisted cough in children with neurological impairment.

\section{Limitations}

Obviously, our study design was suboptimal because of the absence of a control group and lack of randomization. A control group would have been desirable because of the well accepted notion that HFCWC was not shown to be clinically superior to manual chest physical therapy in children with cystic fibrosis. ${ }^{29}$ However, obtaining a control group that is adherent to manual chest physical therapy may be challenging. Yuan et $\mathrm{al}^{22}$ studied HFCWC in children similar to our subjects. They randomized the children into 2 groups, and 7 of the 12 children in the manual chest physical therapy group were $<30 \%$ adherent to manual chest physical therapy, in contrast to excellent adherence in the HFCWC group. Our own clinical experience concurs with that finding. The presence of severe chest wall deformities in many patients with cerebral palsy and neuromuscular impairment makes the delivery of effective manual chest physical therapy nearly impossible. The use of a form-fitting vest to deliver HFCWC overcomes that problem.

Our group of subjects was very heterogeneous; most had cerebral palsy, but other conditions and a wide range of respiratory-compromise severities were involved, and we were unable to factor in all those differences.

We found the use of CoughAssist and the presence of tracheostomy did not affect hospitalization days, but the inclusion of these confounders was post hoc and was not powered for the study outcome measures. Also, we did not monitor our subjects' adherence to the use of the CoughAssist device.

\section{Conclusions}

Long-term HFCWC may reduce the number of hospitalizations and hospitalization days in children with neurological impairment. HFCWC should be considered as an important part of the complex approach to respiratory health in children with neurological impairment and chronic respiratory disorders. Future research efforts should be devoted to further defining the indications for HFCWC in different groups of children with neurological impairment, depending on their diagnosis, cognitive function, and degree of respiratory impairment.

\section{REFERENCES}

1. Birkrant DJ. The assessment and management of the respiratory complications of pediatric neuromuscular diseases. Clin Pediatr 2002; 41(5):301-308.

2. Panitch HB. Respiratory issues in the management of children with neuromuscular disease. Respir Care 2006;51(8):885-895.

3. Seddon PC, Khan Y. Respiratory problems in children with neurological impairment. Arch Dis Child 2003;88(1):75-78.

4. Birnkrant DJ. New challenges in the management of prolonged survivors of pediatric neuromuscular diseases: a pulmonologist's perspective. Pediatr Pulmonol 2006;41(12):1113-1117.

5. Burke RT, Alverson B. Impact of children with medically complex conditions. Pediatrics 2010;126(4):789-90.

6. Burns KH, Casey PH, Lyle RE, Mac Bird T, Fussell JJ, Robbins JM. Increasing prevalence of medically complex children in US hospitals. Pediatrics 2010;126(4):638-646.

7. Yates K, Festa M, Gillis J, Waters K, North K. Outcome of children with neuromuscular disease admitted to paediatric intensive care. Arch Dis Child 2004;89(2):170-175.

8. Simon TD, Berry J, Feudtner C, Stone B, Sheng X, Bratton SL, et al. Children with complex chronic conditions in inpatient hospital settings in the United States. Pediatrics 2010;126(4):647-655.

9. Truog RD, Brock DW, Cook DJ, Danis M, Luce JM, Rubenfeld GD, et al. Rationing in the intensive care unit. Crit Care Med 2006;34(4): 958-963.

10. Panitch HB. Airway clearance in children with neuromuscular weakness. Curr Opin Pediatr 2006;18(3):277-287.

11. Tzeng AC, Bach JR. Prevention of pulmonary morbidity for patients with neuromuscular disease. Chest 2000;118(5):1390-1396.

12. Schramm CM. Current concepts of respiratory complications of neuromuscular disease in children. Curr Opin Pediatr 2000;12(3): 203-207.

13. Miske LJ, Hickey EM, Kolb SM, Weiner DJ, Panitch HB. Use of the mechanical in-exsufflator in pediatric patients with neuromuscular disease and impaired cough. Chest 2004;125(4):1406-1412.

14. Wang CH, Bonnemann CG, Rutkowski A, Sejersen T, Bellini J, Battista V, et al. Consensus statement on standard of care for congenital muscular dystrophies. J Child Neurol 2010;25(12):1559-1581. 


\section{High-Frequency Chest Wall Compression Therapy in Neurologically Impaired Children}

15. Gauld LM. Airway clearance in neuromuscular weakness. Dev Med Child Neurol 2009;51(5):350-355.

16. Birnkrant DJ, Pope JF, Eiben RM. Management of the respiratory complications of neuromuscular diseases in the pediatric intensive care unit. J of Child Neurol 1999;14(3):139-144.

17. Bach JR. Mechanical insufflation/exsufflation: has it come of age? A commentary. Eur Respir J 2003;21(3):385-386.

18. Chatwin M, Ross E, Hart N, Nickol AH, Polkey MI, Simonds AK. Cough augmentation with mechanical insufflation/exsufflation in patients with neuromuscular weakness. Eur Respir J 2003;21(3): 502-508.

19. Reardon CC, Christiansen D, Barnett E, Cabral HJ. Intrapulmonary percussive ventilation vs incentive spirometry for children with neuromuscular disease. Arch Pediatr Adolesc Med 2005;159(6): 526-531.

20. Giarraffa P, Berger KI, Chaikin AA, Axelrod FB, Davey C, Becker B. Assessing efficacy of high-frequency chest wall oscillation in patients with familial dysautonomia. Chest 2005;128(5):3377-3381.

21. Plioplys AV, Lewis S, Kasnicka I. Pulmonary vest therapy in pediatric long-term care. J Am Med Dir Assoc 2002;3(5):318-321.

22. Yuan N, Kane P, Shelton K, Matel J, Becker BC, Moss RB. Safety, tolerability, and efficacy of high-frequency chest wall oscillation in pediatric patients with cerebral palsy and neuromuscular diseases: an exploratory randomized controlled trial. J Child Neurol 201:25(7): 815-821.

23. Warwick WJ, Hansen LG. The long-term effect of high-frequency chest compression therapy on pulmonary complication of cystic fibrosis. Pediatr Pulmonol 1991;11(3):265-271.

24. King PT, Daviskas E. Pathogenesis and diagnosis of bronchiectasis. Breathe 2010;6:342-351.

25. Stafler P, Carr SB. Non-cystic fibrosis bronchiectasis: its diagnosis and management. Arch Dis Child Educ Pract Ed 2010;95(3):73-82.

26. Piccione JC, McPhail GL, Fenchel MC, Brody AS, Boesch RP. Bronchiectasis in chronic pulmonary aspiration: risk factors and clinical implications. Pediatr Pulmonol 2012;47(5):447-452.

27. Kluft J, Beker L, Castagnino M, Gaiser J, Chaney H, Fink RJ. A comparison of bronchial drainage treatments in cystic fibrosis. Pediatr Pulmonol 1996;22(4):271-274.

28. Willis LD, Warren RH. Acute hypoxemia in a child with neurological impairment associated with high frequency chest wall compression. Respir Care 2007;52(8):1027-1029.

29. Arens R, Gozal D, Omlin KJ, Vega J, Boyd KP, Keens T, et al. Comparison of high frequency chest compression and conventional chest physiotherapy in hospitalized patients with cystic fibrosis. Am J Respir Crit Care Med 1994;150(4):1154-1157. 